\title{
Analysis of Data Warehouse for Transactional Analysis Methods Online Analytical Processing (OLAP) at Company XYZ Silver
}

\author{
Putu Widiadnyana ${ }^{[1]}$, M. Azman Maricar ${ }^{[2]}$, I Nyoman Arnawan ${ }^{[3]}$, and Sri Ariyani ${ }^{[4]}$ \\ [1][2][3] Department of Electrical and Computer Engineering, Post Graduate Program, Udayana University \\ [4] Department of Electrical and Computer Engineering, Udayana University \\ E-Mail: widiadnyana11@gmail.com
}

\begin{abstract}
Utilization of information technology is a necessity in looking at opportunities available for decision making by the management. With the ability of information technology to analyze existing used the data into useful information for a company. OLAP is Able to Overcome problems in the data processing mechanisms guided to know various information from different angles. By utilizing transactional analysis, then we know the reaction of customers in choosing the products that we will market. The result of Web-based sales has Decreased for the Gianyar and Denpasar regions but has Increased for the Ubud and online areas so as to give a benefit in terms of production costs. With the training for a sales increase of sales can evenly across locations and need input from customers related to minimize returns.
\end{abstract}

\section{Keywords-OLAP; Sales; Transactional Analysis}

\section{INTRODUCTION}

The information technology has been a necessity in view of the opportunities that exist for decision-making by management. With the ability to use information technology is able to analyze the data into useful information for a company. Various information is data able to be processed using software existingbut in utilizing information technology in need of expertise in using a variety ofsoftware existingthat allows users to access information. OLAP is able to overcome the problems in the data processing mechanisms are guided to know various information from various viewpoints.

\section{LITERATURE REVIEW}

\section{A. Data Warehouse}

Data warehouse is data that a very large that have a natureoriented subject, integrated, time-variant, and is fixed on the stored data in support of decision-making process management, which can improve the accuracy and quality of information that is easily understandable and can be accesed easily by pemkaai containing desired information [1]. There are four characteristics possessed by the data warehouse is as follows.

1. Oriented on the subject
Data warehouse that is designed to analyze the data, based on certain subjects that contain information in an organization that is important forprocessing. Decision support for example, sales data, customer, and so on.

2. Integration

Data warehouse is derived from data that is different and separate but can be stored in the same format and integrated with one another

3. Range of time

Data warehouse is the data that is accurate and valid at any given time which is useful for estimating or comparing information

4. Non-volatile

Data warehouse is not always updatedin real time [1,2].

\section{B. Transactional Analysis}

TheTransactional analysis is one of a new way of determining the type of prospective buyers as well as determining sales strategy. The concept is very simple and dpat sderhana to be understood and used as models to improve communication. Everyone has self conditions that determine the person's mental attitude in the message communication and behavior. the condition itself is a condition that affects the behavior of both prospective buyers and salesmen berrati is a system that encourages a feeling of a certain thought patterns consistently [6].

\section{Online Analytical Processing (OLAP)}

OLAP is a method used to analyze the data to be made a report that summarizes the relationship anatara data and information that can be utilized by the user in a fast, consistent, and interktif $[2,4]$. There are several advantages to using OLAP namely:

1. Users do not need to know SQL language

2. Users do not need to know the relational database model

3. Able to improve query performance and improve system scalability 
4. Enhancing existing systems in the calculation into the information

5. Management system that is easier

6. To streamline the cost of data maintenance

7. Reducing and freeing load data warehouse

8. More centralized control of analytical data [5].

\section{METHODOLOGY}

\section{A. Data Analysis}

Various data have been obtained further analyzed to obtain the appropriate attributes in the manufacture of a data warehouse. The results of this analysis will be used to determine the dimensions, facts, and an appropriate schema for the data model warhouse..

\section{B. Phase Extraction}

Data that will be used in the design of data warehouse

\section{Data Warehouse Design}

Data to be used as data storage that has been transformed. Then the data entered into the data warehouse to be used.

\section{OLAP Design Stage}

OLAP allows the user to be able to see the data with different dimensions due to the used data that differ from one another.

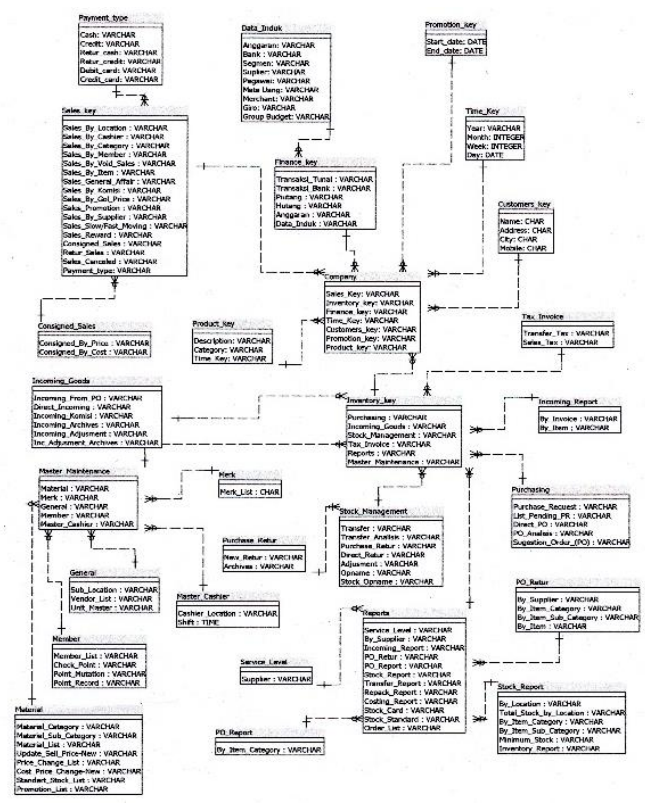

Fig. 1. Model of Data for Sales Performance Analysis

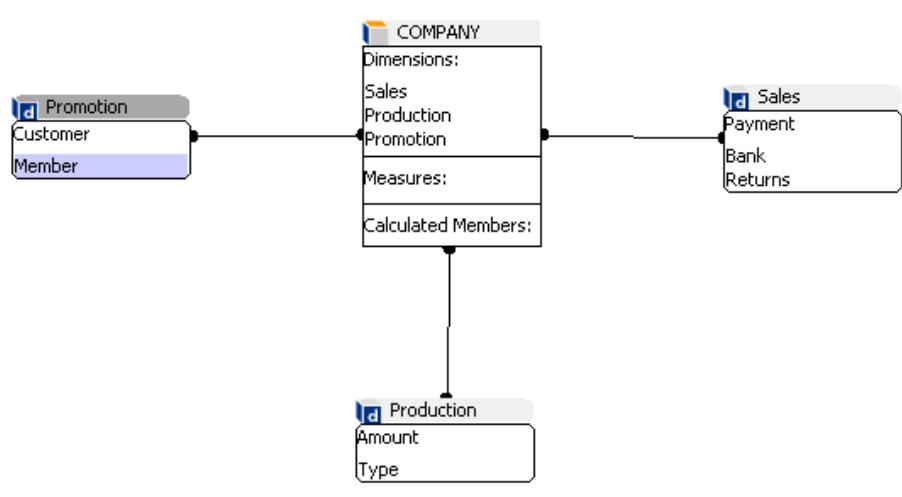

Fig. 2. Data Model for Data Warehouse

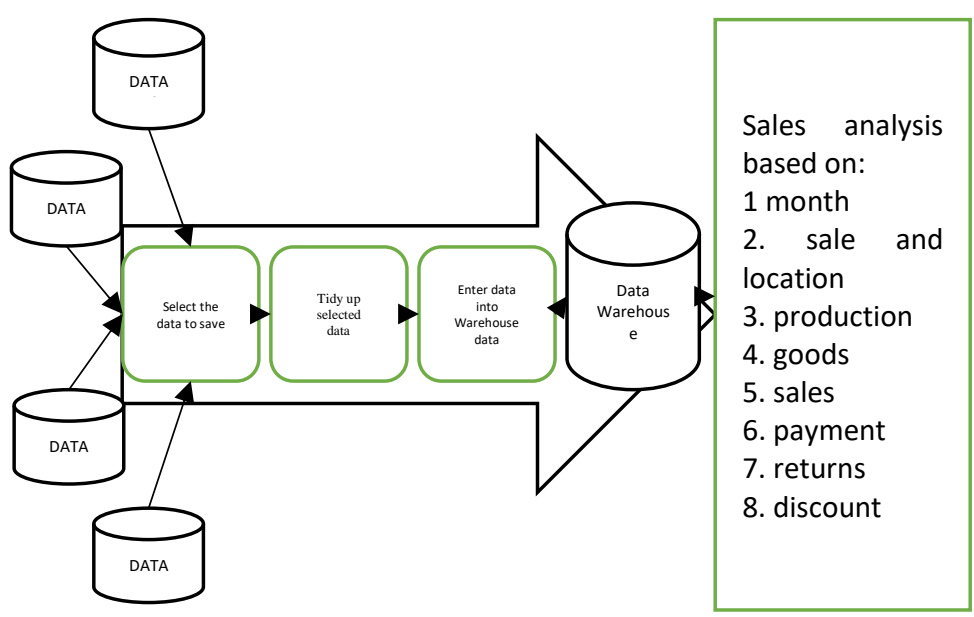

Fig. 3. Architecture Design Data Warehous

\section{RESULT ANALYSIS}

Here are results of some analysis of the data warehouse that is obtained as follows.

Table 1 Sales Analysis (Month and Location)

\begin{tabular}{|c|c|c|c|}
\hline LOCATION & JANUARY & FEBRUARY & MARCH \\
\hline GIANYAR & Rp $110,325,164$ & $\begin{array}{l}\mathrm{Rp} \quad 51,729,525 \\
\end{array}$ & $\begin{array}{ll}\mathrm{Rp} & 81,733,556 \\
\end{array}$ \\
\hline UBUD & Rp $15,000,000$ & $25,088,000$ & $26,831,000$ \\
\hline DENPASAR & $\mathrm{Rp} \quad 84,429,000$ & Rp $\quad 78,419,209$ & $\mathrm{Rp} \quad 26,831,000$ \\
\hline ONLINE & Rp $\quad 2,496,000$ & Rp $\quad 7,672,025$ & $16,433,000$ \\
\hline TOTAL & Rp $212,250,164$ & Rp $162,908,759$ & Rp $151,828,556$ \\
\hline
\end{tabular}


PENJUALAN LOKASI

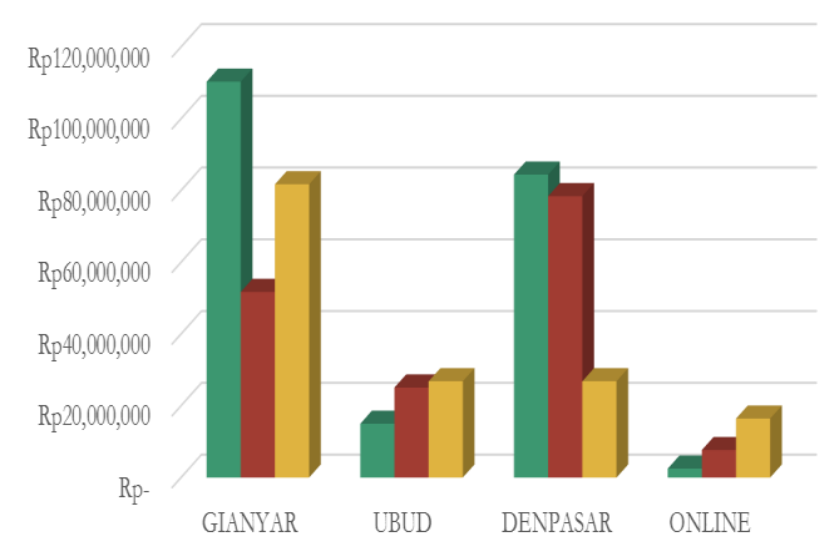

-JANUARI -FEBRUARI M MARET

Fig. 4. Sales Analysis (Month and Location)

Table 2 Sales Analysis (Production)

\begin{tabular}{|c|c|c|c|}
\hline BULAN & $\begin{array}{c}\text { TOTAL } \\
\text { JUMLAH } \\
\text { BARANG }\end{array}$ & $\begin{array}{c}\text { BERAT SETOR } \\
\text { BERSIH }\end{array}$ & $\begin{array}{c}\text { TOTAL HARGA } \\
\text { POKOK }\end{array}$ \\
\hline DESEMBER & 610 & 1,968 & Rp $137,414,643$ \\
\hline JANUARI & 604 & 1,735 & Rp $128,470,000$ \\
\hline FEBRUARI & 437 & 923 & Rp $105,155,000$ \\
\hline MARET & 825 & 2,510 & Rp $136,081,000$ \\
\hline
\end{tabular}

\section{TOTAL HARGA POKOK PRODUKSI}

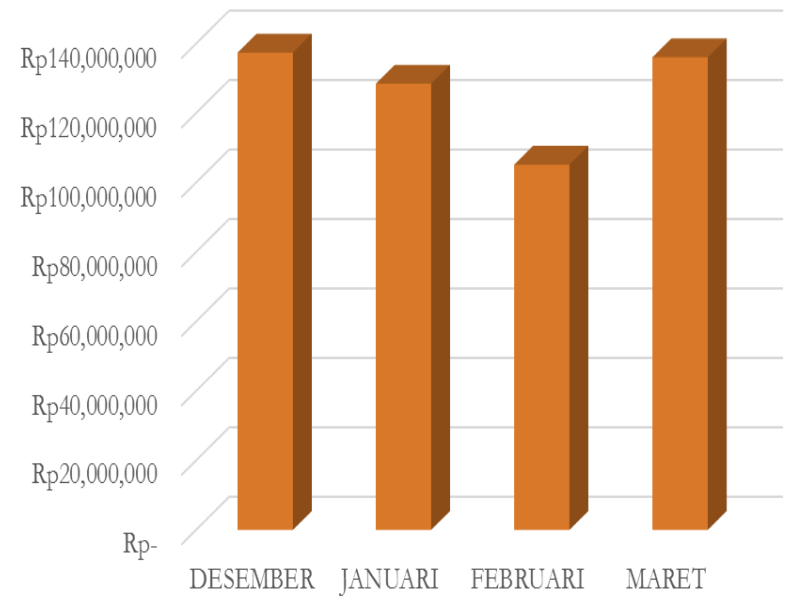

Fig. 5. analysis of Sales (Production )
Table 3 Analysis of Sales Revenue (Sales)

\begin{tabular}{|c|c|c|c|}
\hline NAME & JANUARY & FEBRUARY & MARCH \\
\hline SALES A & $\mathrm{Rp} \quad 23,166,000$ & $\operatorname{Rp} \quad 25,906,000$ & $\mathrm{Rp} \quad 12,837,000$ \\
\hline SALES B & $\mathrm{Rp} \quad 5,512,009$ & $\mathrm{Rp} \quad 9,512,009$ & Rp $19,881,000$ \\
\hline SALES C & $\mathrm{Rp} \quad 58,831,000$ & Rp $\quad 37,333,200$ & $\mathrm{Rp} \quad 10,320,000$ \\
\hline SALES D & $\mathrm{Rp} \quad 33,200,000$ & Rp $\quad 25,088,000$ & $\mathrm{Rp} \quad 21,026,000$ \\
\hline SALES F & $\mathrm{Rp} \quad 23,000,000$ & $\mathrm{Rp} \quad 43,189,525$ & $\mathrm{Rp} \quad 45,274,556$ \\
\hline SALES G & $\mathrm{Rp} \quad 20,816,000$ & $\mathrm{Rp} \quad 14,208,000$ & $\mathrm{Rp} \quad 10,636,000$ \\
\hline SALES H & $\begin{array}{c}\mathrm{Rp} \\
-\end{array}$ & $\begin{array}{l}\mathrm{Rp} \\
-\end{array}$ & $3,800,000$ \\
\hline SALES I & $\begin{array}{c}\mathrm{Rp} \\
- \\
\end{array}$ & $\begin{array}{c}\mathrm{Rp} \\
- \\
\end{array}$ & $5,816,000$ \\
\hline
\end{tabular}

PENJUALAN SALES

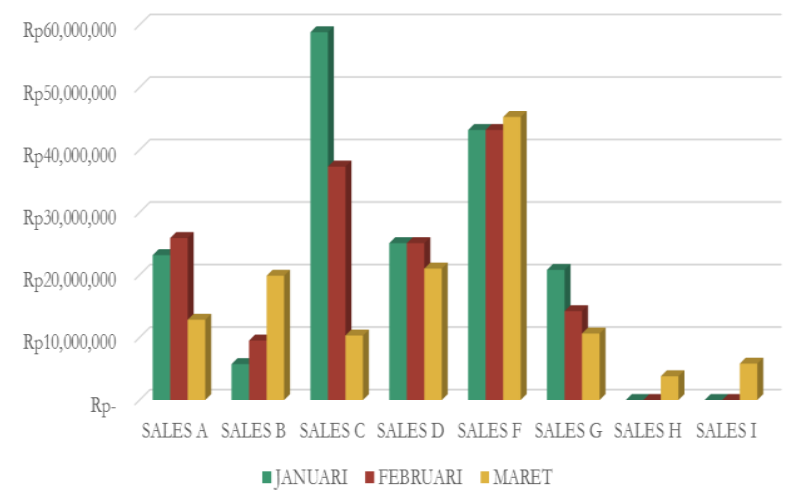

Fig. 6. Analysis of Sales Revenue (Sales)

Table 4 Analysis of Sales (Payments))

\begin{tabular}{|c|c|c|c|}
\hline BULAN & CASH & $\begin{array}{c}\text { CREDIT } \\
\text { CARD }\end{array}$ & DEBIT CARD \\
\hline & $\mathrm{Rp}$ & $\mathrm{Rp}$ & $\mathrm{Rp}$ \\
JANUARI & $39,155,000$ & $43,837,009$ & $44,130,000$ \\
\hline & $\mathrm{Rp}$ & $\mathrm{Rp}$ & $\mathrm{Rp}$ \\
FEBRUARI & $37,042,200$ & $47,262,009$ & $32,126,000$ \\
\hline & $\mathrm{Rp}$ & $\mathrm{Rp}$ & $\mathrm{Rp}$ \\
MARET & $34,421,000$ & $44,184,000$ & $15,496,000$ \\
\hline
\end{tabular}

\section{PEMBAYARAN}

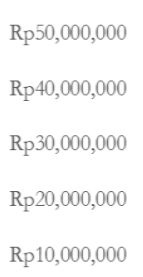

Rp-

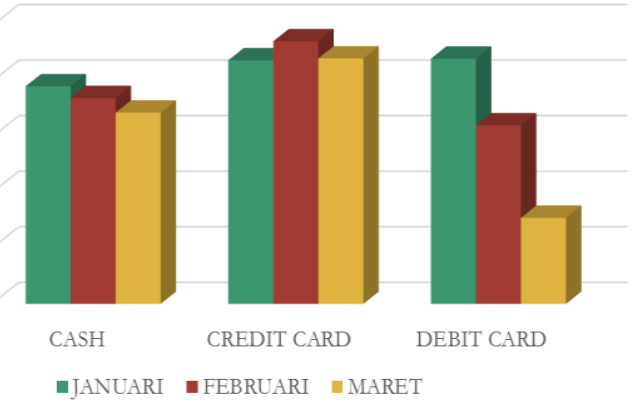


Fig. 7. Sales Analysis (Payments)

Table 5 Sales analysis (Return)

\begin{tabular}{|c|c|c|}
\hline JANUARI & FEBRUARI & MARET \\
\hline Rp $\quad 2,258,000$ & Rp $\quad 1,072,000$ & $1,787,600$ \\
\hline
\end{tabular}

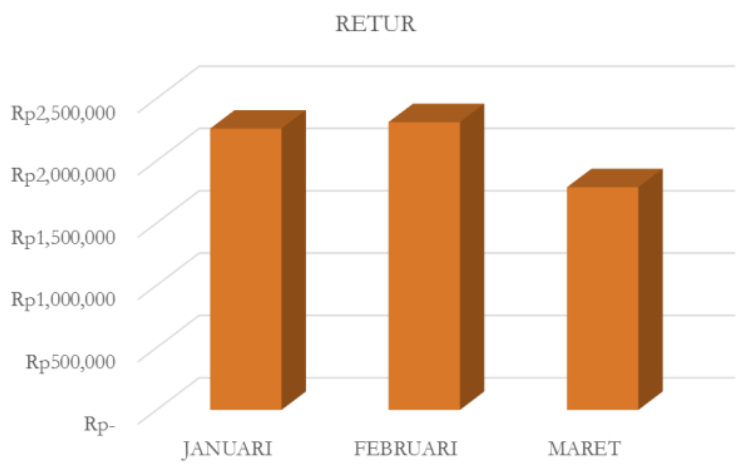

Fig. 8. analysis of Sales (Sale)

Table 6 Sales analysis (Discounts)

\begin{tabular}{|c|c|c|}
\hline JANUARI & FEBRUARI & MARET \\
\hline Rp & & \\
$147,219,529$ & $\operatorname{Rp~} 124,017,451$ & $\operatorname{Rp~} 133,838,167$ \\
\hline
\end{tabular}

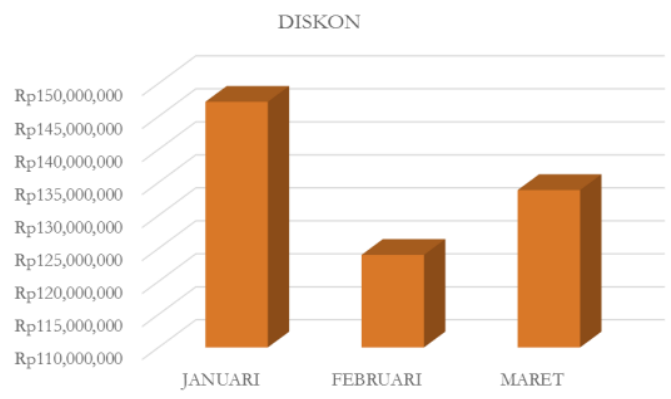

Fig. 9. Analysis of Sales (Sale)

\section{CONCLUSION}

It can be concluded that the results of decreased sales by location for the area of gianyar and denpasar but increased to the area of ubud and online so that they can benefit by cost of production. With the training for sales to increase sales are evenly distributed throughout the location and the need for customer feedback related to minimize returns..

\section{REFERENCES}

[1] Ditya Octavianto dan Tutut Wurijanto. Pengolahan Data Warehouse Terhadap Sumber Data Akademik Dan Kemahasiswaan Stikom Sebagai Sistem Pendukung Keputusan Surabaya. Prosiding Seminar Nasional Sistem \& Teknologi Informasi (SNASTI), 22 Agustus 2007.

[2] Budi Santosa, Dessyanto Boedi, dan Markus Priharjanto. Analisa Data Transaksional Pada E-Commerce Denganteknologi Olap (On-Line Analytical Process). Seminar Nasional Informatika 2011 (semnasIF 2011) UPN "Veteran" Yogyakarta, 2 Juli 2011; ISSN : 1979-2328.

[3] Randy Oktrima Putra. Rancang Bangun Data Warehouse Untuk Analisis Kinerja Penjualan Pada Industri Dengan Model Spa-Dw (Sales Performance Analysis - Data Warehouse) (studi kasus : PT. Semen Padang). Tesis, Universitas Diponegoro; 2012.

[4] Maimunah, Siti Farhatus, dan Randy Andrian. Rancang Bangun Aplikasi Data Warehouse Untuk Business Intelligence. CSRID Journal, Vol.4 No.1 Februari 2012, Hal. 27 - 36.

[5] Slamet Kacung dab Lambang Probo. Rancangan dan Pembuatan Data Warehouse untuk Kebutuhan Sistem Pendukung Keputusan (Studi Kasus: Departemen Marketing dan Komunikasi Universitas Dr Soetomo Surabaya. Jurnal.

[6] R. Dwi Anggiadi H. Pengaruh Atribut Produk Jaket Star Seeker Terhadap Keputusan Pembelian Konsumen di Bandung. Skripsi.

[7] Armadyah Amborowati. 2008. Perancangan Dan Pembuatan Data Warehouse Pada Perpustakaan Stmik Amikom Yogyakarta. Seminar Nasional Aplikasi Sains dan Teknologi 2008 - IST AKPRIND Yogyakarta

[8] Fatah Yasin Al Irsyadi . 2014. Implementasi Data Warehouse Dan Data Mining Untuk Penentuan Rencana Strategis Penjualan Batik (Studi Kasus Batik Mahkota Laweyan). KomuniTi, Vol. VI, No. 1 Maret 2014

[9] Tanty Oktavia . 2011. Perancangan Model Data Warehouse Dalam Mendukung Perusahaan Jasa Pengiriman. Seminar Nasional Informatika 2011 (semnasIF 2011) UPN "Veteran" Yogyakarta, 2 Juli 2011

[10] Lita Alfriany Ndoloe. 2012. Sistem Informasi Lulusan Dengan Metode Online Analitycal processing (OLAP) Pada Politeknik Negeri Kupang. Jurnal Sistem Informasi Bisnis

[11] Novi Sofia Fitriasari. 2008. Perancangan Sistem Informasi Business Intelegence Lulusan Dengan Menerapkan Metode Olap. Seminar Nasional Aplikasi Teknologi Informasi 2008 (SNATI 2008) Yogyakarta, 21 Juni 2008

[12] Dimara Kusuma Hakim. 2011. Implementasi Online Analytical Processing (Olap) Pada Studi Kasus Sistem Informasi Manajemen Perijinan Menggunakan Alat Bantu Microsoft Business Intelligence Development Studio. Techno, Volume 12 No. 1, April 2011, ISSN 1410 $-8607$

[13] Zainal Arifin. 2013.Rancang Bangun Sistem Business Intelligence Universitas Sebagai Pendukung Pengambilan Keputusan Akademik. Jurnal Sistem Informasi Bisnis 01(2013)

[14] Fadhil Husni Putra. 2016. Aplikasi Data Warehouse Dan On-Line Analytical Processing (Olap) (Studi Kasus: Permintaan Dan Pemakaian Obat Di Puskesmas Poasia Kota Kendari). SemanTIK, Vol.2, No.2, JulDes 2016, pp. 1-12 ISSN : 2502-8928

[15] Adi Supriyatna. 2016. Sistem Analisis Data Mahasiswa Menggunakan Aplikasi Online Analytical Processing (Olap) Data Warehouse. Jurnal Pilar Nusa Mandiri Vol.XII, No. 1 Maret 2016 